\title{
Effects of Rotating Magnetic Islands Driven by External Perturbation Fields in the TU-Heliac
}

\author{
Sumio KITAJIMA, Hajime UMETSU, Mamiko SASAO, Atsushi OKAMOTO, \\ Takashi KOBUCHI, Yutaka TANAKA, Hiroyasu UTOH, Hiroshi AOYAMA, Keisuke IWAZAKI, \\ Shigeru INAGAKI ${ }^{1)}$, Masakazu TAKAYAMA ${ }^{2)}$, Akio KOMORI ${ }^{3)}$, Kiyohiko NISHIMURA ${ }^{3)}$, \\ Yasuhiro SUZUKI ${ }^{3)}$, Masayuki YOKOYAMA ${ }^{3)}$, Hiromi TAKAHASHI ${ }^{3)}$ \\ Department of Quantum Science and Energy Engineering, Tohoku University, Sendai 980-8579, Japan \\ ${ }^{1)}$ Research Institute for Applied Mechanics Kyushu University, 6-1 Kasuga-kouen, Kasuga, Fukuoka 816-8580, Japan \\ 2) Akita Prefectural University, Honjyo, Akita 015-0055, Japan \\ ${ }^{3)}$ National Institute for Fusion Science, 322-6 Oroshi-cho, Toki 509-5292, Japan
}

(Received 16 November 2007 / Accepted 5 March 2008)

\begin{abstract}
A new method for rotating magnetic islands by external perturbation fields is proposed. In the experiments, perturbation fields were produced using four pairs of cusp field coil, in which alternating currents having a $\pi / 2$ phase shift flowed. A phase shifter for the coil currents was designed and constructed. The phase difference in the floating potential signals measured using two Langmuir probes confirmed that the magnetic islands rotated in the counterclockwise direction $(\mathrm{c} / \mathrm{cw})$. The clockwise $(\mathrm{cw})$ rotation was also observed in the plasma biased by the hot cathode electrode. These experimental results suggest the ability of producing plasma poloidal rotation driven by rotating islands.
\end{abstract}

(C) 2008 The Japan Society of Plasma Science and Nuclear Fusion Research

Keywords: stellarator, heliac, magnetic island, poloidal rotation, perturbation field, electrode bias.

DOI: $10.1585 /$ pfr.3.S1027

\section{Inroduction}

A study on the effects of magnetic islands on transport in helical devices is important, because it leads to the advanced control method for a plasma periphery in a helical fusion reactor. The magnetic island effects on transport have been surveyed widely in the Large Helical Device (LHD) [1-6]. For the research on the island effects on the confinement modes, the Tohoku University Heliac (TU-Heliac) has advantages that (1) the position of a rational surface is changeable by selecting the ratio of coil currents, (2) the island formation can be controlled by external perturbation field coils, and (3) a radial electric field and particle transport can be controlled by electrode biasing. In the TU-Heliac, the improved mode transition was triggered by electrode biasing using a hot cathode composed of $\mathrm{LaB}_{6}$. The driving force $\boldsymbol{J} \times \boldsymbol{B}$ for the plasma poloidal rotation was externally controlled, and the poloidal viscosity was successfully estimated from the external driving force [7-9]. In recent experiments, the ion viscosity in the biased plasma with islands was roughly estimated. It suggested that the ion viscosity increased with the magnetic island width [10]. Therefore, it is expected that the plasma poloidal rotation will be driven by the poloidal rotation of the island. The purposes of this experiment are to propose the new method of rotating islands by the external perturbation fields, survey the ability of the plasma poloidal rota-

author's e-mail: sumio.kitajima@qse.tohoku.ac.jp tion driven by rotating islands, and study the rotating island effects on confinement modes in TU-Heliac.

\section{Experimental Setup}

\subsection{TU-Heliac}

The TU-Heliac is a four-period heliac (major radius, $0.48 \mathrm{~m}$; average plasma radius, $0.07 \mathrm{~m}$ ). The heliac configurations were produced using three sets of magnetic field coils; 32 toroidal field coils, a center conductor coil, and one pair of vertical field coils, as shown in Fig. 1 (a). Three capacitor banks, consisting of two-stage pulse forming networks, separately supplied coil currents of $10 \mathrm{~ms}$ flat top [11]. The target plasma for external perturbation fields was He plasma produced by low-frequency joule heating ( $\left.f=18.8 \mathrm{kHz}, P_{\text {out }} \sim 35 \mathrm{~kW}\right)$. The joule heating power was supplied to one pair of the poloidal coils wound outside the toroidal coils [12]. The vacuum vessel was filled with fueling neutral He gas, and was sealed from the evacuation system before every discharge. The electron density and temperature measured using a Langmuir probe (triple probe) were $\sim 6 \times 10^{17} \mathrm{~m}^{-3}$ and $\sim 20 \mathrm{eV}$ at the magnetic axis, respectively, and the magnetic field strength at the axis was $0.3 \mathrm{~T}$.

\subsection{External perturbation coils}

In the TU-Heliac, we selected a current ratio to locate a rational flux surface $(n / m=5 / 3)$ in the plasma pe- 

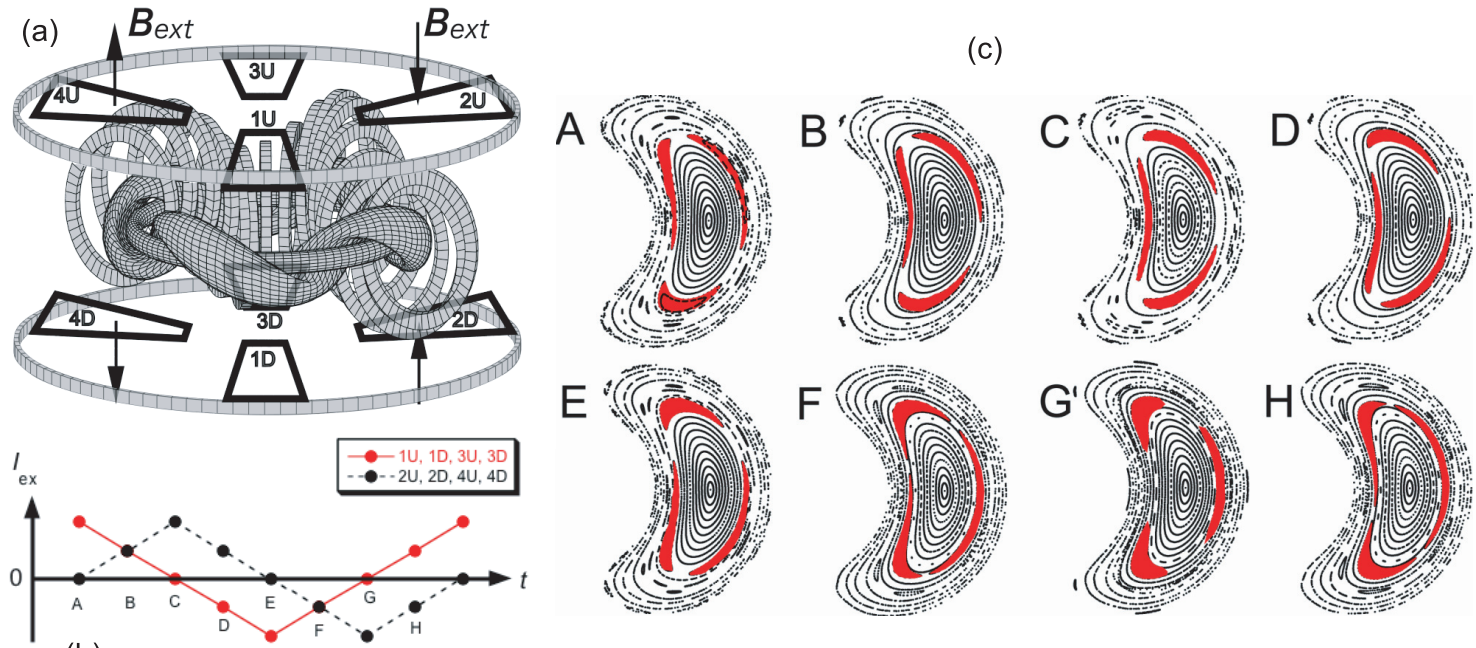

(b)

Fig. 1 (a) Bird's eye-view of TU-Heliac, (b) time evolution of the currents in both groups of coils, (c) magnetic surfaces corresponding to each typical time (A-H in (b))

riphery. An efficient configuration of the perturbation coils for generating islands $(m=3)$ was investigated. We decided 4 pairs of upper and lower external perturbation field coils, located at the toroidal angle $\phi=0^{\circ}, 90^{\circ}, 180^{\circ}$, and $270^{\circ}$, which generated a cusp field at each toroidal angle, as shown in Fig. 1 (a). We explored the possibility of the poloidal rotation of islands by changing the phase of each perturbation coil current. Our employed method consisted of dividing perturbation coils into two groups (the first group consists of $1 \mathrm{U} 1 \mathrm{D}$ and 3U3D, and the second group of 2U2D and 4U4D) and changing the perturbation current separately; and one group's current was $I_{\mathrm{ex}}=I_{0} \sin (\omega t)$ and other was $I_{\mathrm{ex}}=I_{0} \sin (\omega t-\pi / 2)$, as shown in Fig. 1 (b). Each coil produces an alternating cusp field with the frequency $\omega$. Figure 1 (c) shows the magnetic surfaces corresponding to the typical time (A-H). The result of magnetic surface calculations suggested that islands rotate in the poloidal direction with a poloidal velocity of $\langle r\rangle \omega / m$. Here, $\langle r\rangle$ is the average radius of the rational flux surface $(n / m=$ $5 / 3$ ), and $m$ is the mode number of the magnetic islands. The poloidal rotation velocity can be changed using the frequency $\omega$. Furthermore, we can select the poloidal rotation direction (clockwise $\mathrm{cw}$ or counterclockwise direction $\mathrm{c} / \mathrm{cw}$ ) by changing the polarity of the phase shift. Here, the $\mathrm{c} / \mathrm{cw}$ direction means the ion diamagnetic direction.

In the experiment, we adopted the phase shifter shown in Fig. 2, which consisted of precisely tuned capacitors and a resistor. The perturbation field coil current was $1.2 \mathrm{kA}$ turn, and the frequency was $2.515 \mathrm{kHz}$. The current and frequency were selected to perform the preliminary experiments, and we planned to increase the current and frequency. Figure 3 shows the perturbation coil currents measured using the Rogowski coils. We can see that the two group coil currents have a $\pi / 2$ phase shift and the same values. The $m=3$ magnetic islands rotate due to the alter-

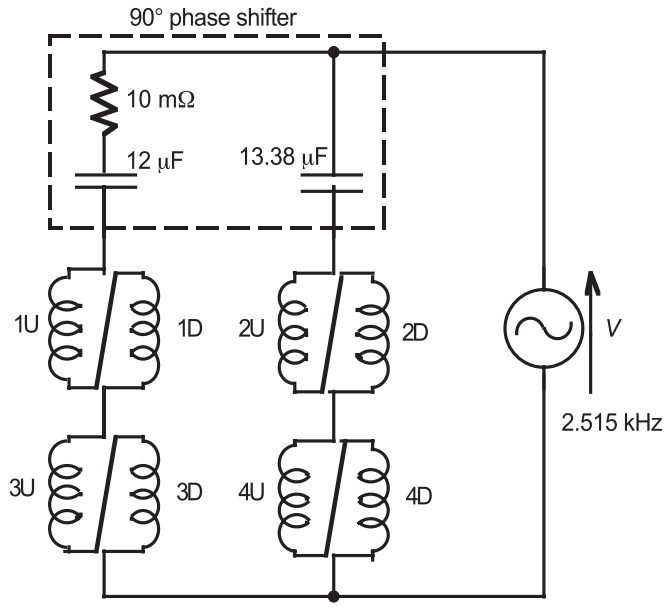

Fig. 2 Schematic circuit for the external perturbation coils.

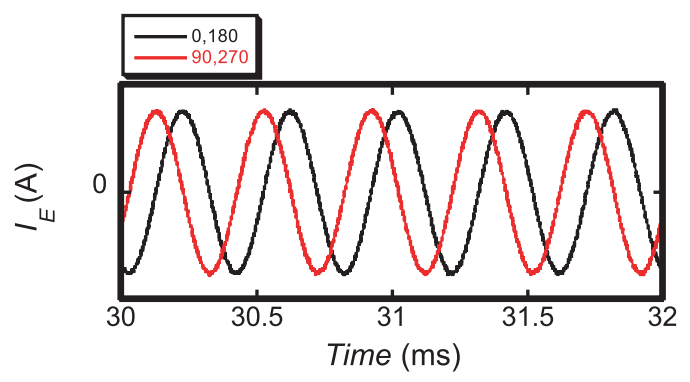

Fig. 3 Perturbation coil currents measured by the Rogowski coils. Two group coil currents have $\pi / 2$ phase shift and same values.

nating perturbation fields, as shown in the next section.

Figure 4 shows the magnetic surfaces with $m=3$ magnetic islands, which were produced by the external perturbation fields. The $m=3$ magnetic islands rotated by the 


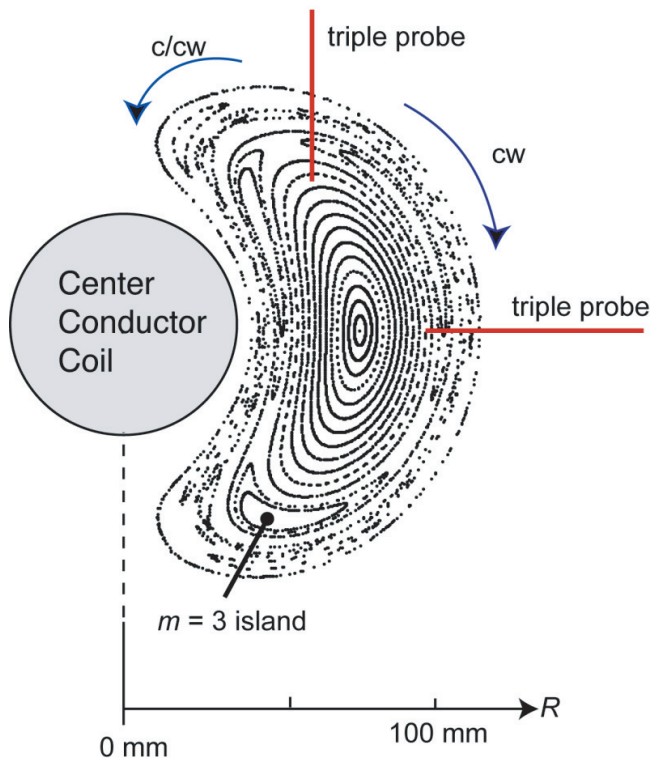

Fig. 4 Cross section of magnetic surfaces with $m=3$ magnetic islands. Two Langmuir probes on the magnetic surface at the positions which were separated about a half of the poloidal length of the island.

alternating perturbation fields.

\section{Phase Shift in Probe Measurements}

To investigate experimentally the effect of the external perturbation field, we measured the floating potential using a Langmuir probe (high-speed triple probe [13,14]), which was inserted from the low-field side at the toroidal angle $\phi=0^{\circ}$. In Fig. 5, it is clear that the floating potential signal (lower trace) has the frequency component of the perturbation fields and the phase shift to the external perturbation coil current (upper trace). Then, we measured the radial profile of the FFT power spectrum in the floating potential signals. Figure 6 shows the relation between the power spectrum of the floating potential and the radial position of the Langmuir probe. Figure 6 clearly shows that the frequency of the perturbation field coil current $(f$ $=2.515 \mathrm{kHz}$ ) was excited around the $m=3$ magnetic island. Low-frequency fluctuations (between 0 and $1.2 \mathrm{kHz}$ ) were observed in the target plasma with/without the external perturbation field.

In order to confirm the rotation of the magnetic islands, we set the two Langmuir probes on the magnetic surface at positions separated by about a half of the poloidal length of the island. These probes were set at the same meridian plane at the toroidal angle $\phi=0^{\circ}$, as shown in Fig. 4. We measured the phase shift in the frequency of the perturbation fields $(f=2.515 \mathrm{kHz})$ between the two probe signals. Figure 7 shows the difference in the phase between the floating potential signals at the three radial points. The open and closed symbols correspond to the rotation of the islands in the $\mathrm{cw}$ and $\mathrm{c} / \mathrm{cw}$ directions, respectively, as expected from the calculation. In the $m=3$

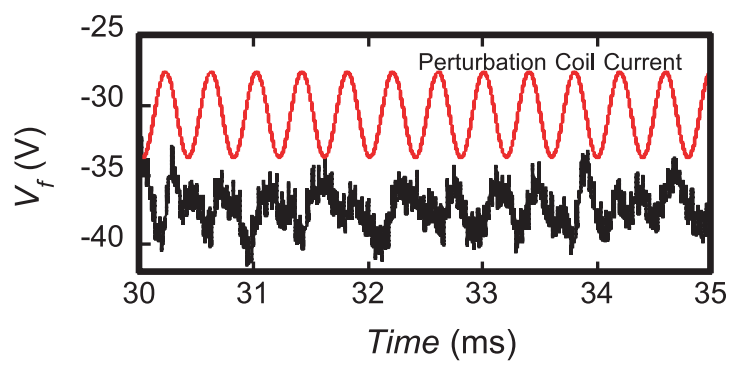

Fig. 5 External perturbation coil current and the floating potential measured by a Langmuir probe (high speed triple probe).

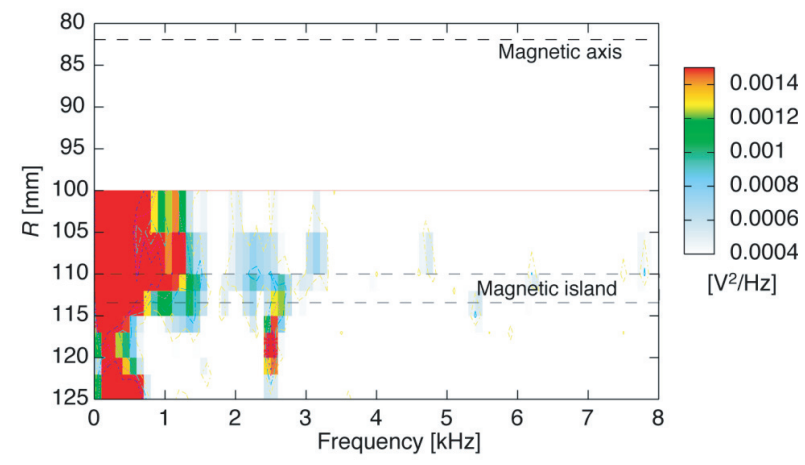

Fig. 6 Relation between the FFT power spectrum of floating potential and the radial position of the Langmuir probe.

islands region at $R=105 \mathrm{~mm}$, the measured phase difference was about $\pi$, which corresponded to a rotation in the $\mathrm{c} / \mathrm{cw}$ direction with the velocity $v \sim 0.2 \mathrm{~km} / \mathrm{s}$. This experimental result suggests that the rotating islands have the ability to drive the plasma poloidal rotation. However, in the $\mathrm{cw}$ case, the phase difference had small positive values, which were inconsistent with the calculation, because they should be having a value of $-\pi$. In these experiments, the target plasma had weak positive radial electric fields $\left(E_{\mathrm{r}} \sim 2 \mathrm{kV} / \mathrm{m}\right)$; therefore, the bulk plasma rotated in the c/cw direction by the $\boldsymbol{E} \times \boldsymbol{B}$ rotation. Therefore, we can infer that there are some restrictions on the rotation of the islands in the direction, which is opposite to the natural direction of the bulk plasma rotation (c/cw direction).

In the TU-Heliac, we can bias the potential to the plasma using the hot cathode electrode composed of $\mathrm{LaB}_{6}$. We can control the negative radial electric field externally by biasing the electrode. The hot cathode (diameter, $10 \mathrm{~mm}$; length, $17 \mathrm{~mm}$ ) was inserted horizontally into the plasma from the low-magnetic field side at the toroidal angle $\phi=270^{\circ}$. The $m=3$ islands were formed at the outside of the hot cathode. The direction of the $\boldsymbol{E} \times \boldsymbol{B}$ rotation was in the cw direction, since $\boldsymbol{E}$ was the radial electric field produced by the biasing. Thus, we can decelerate the natural $\mathrm{c} / \mathrm{cw}$ plasma rotation. We attempted to apply the external perturbation fields to rotate the islands in the biased plasma. Figure 8 shows the relation between the phase 


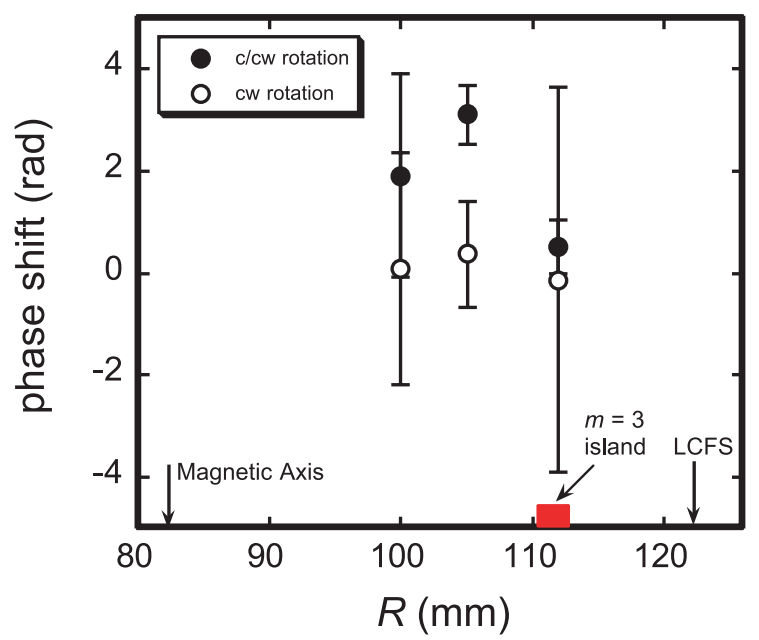

Fig. 7 Difference of the phase between the floating potential signals at the three radial points. The open symbols and closed symbols denote the $\mathrm{cw}$ and $\mathrm{c} / \mathrm{cw}$ directions of the rotating islands which are expected by the calculations.

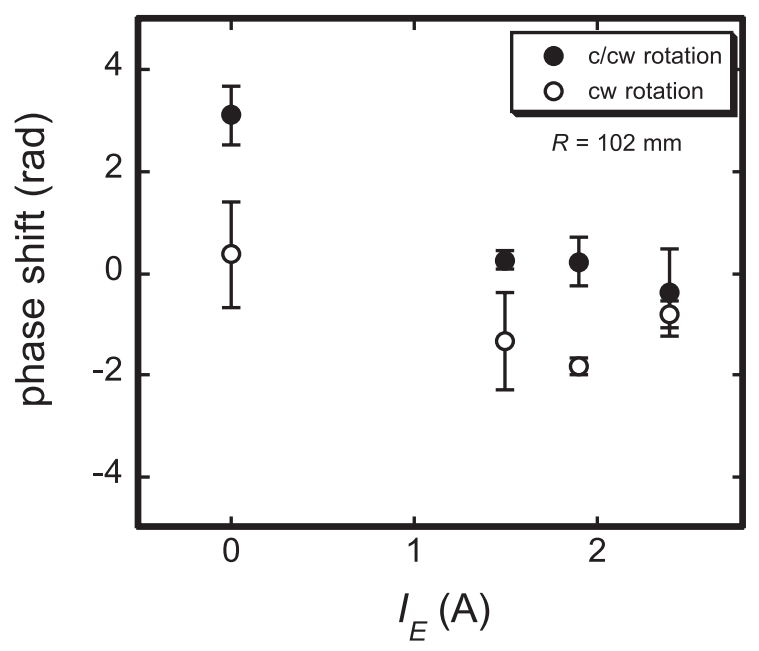

Fig. 8 Relation between the differences of the phase in two floating potential signals and the electrode current. The increase in the electrode current means the increase in the velocity of the cw bulk plasma rotation. The data at $I_{\mathrm{E}}=$ 0 were measured at $R=105 \mathrm{~mm}$.

differences between two floating potential signals and the electrode current used for plasma biasing. An increase in the electrode current indicates an increase in the cw plasma rotation velocity. We showed the results without electrode biasing $\left(I_{\mathrm{E}}=0 \mathrm{~A}, R=105 \mathrm{~mm}\right)$ shown in Fig. 7. Before the application of the external perturbation fields, we measured the velocity of the plasma poloidal rotation by the phase velocity of high-frequency fluctuations in the floating potential $[8,15]$. The result was that the plasma rotation vanished when the electrode current was about $2 \mathrm{~A}$ without the external perturbation fields. In Fig. 8, we can see that in the $\mathrm{cw}$ island rotation case, the phase difference was $-2 \mathrm{rad}$ at $I_{\mathrm{E}}=2 \mathrm{~A}$, which was consistent with the calculations and suggested the rotation of the magnetic island, although in the $\mathrm{c} / \mathrm{cw}$ island rotation cases, the phase difference decreased compared with the experimental results in Fig. 7. We infer that the bulk plasma rotation with the external perturbation fields was overdriven by the electrode current of $I_{\mathrm{E}} \sim 2 \mathrm{~A}$, because the plasma parameters changed with/without the external perturbation fields. In the external $\boldsymbol{E} \times \boldsymbol{B}$ rotation experiments, the radial electric field was formed by the transition phenomena by biasing. Therefore, the external $\boldsymbol{E} \times \boldsymbol{B}$ rotation cannot be controlled smoothly by biasing. We infer that the bulk plasma rotation with the external perturbation fields vanishes in the condition of $I_{\mathrm{E}} \sim 0.8 \mathrm{~A}$. For a higher value of the electrode current $\left(I_{\mathrm{E}} \sim 2.3 \mathrm{~A}\right)$, the phase difference in the $\mathrm{cw}$ direction decreased, which implies that the islands would be destroyed by the external $\boldsymbol{E} \times \boldsymbol{B}$ rotation.

\section{Summary}

We proposed a method for rotating the magnetic islands using external perturbation fields, which were produced using four pairs of cusp fields. The alternating currents for the perturbation coils have a $\pi / 2$ phase shift. We designed and constructed the phase shifter for the coil currents, and measured the phase difference in the floating potential signals using two Langmuir probes, which confirmed the rotation at the inside of the magnetic islands in the $\mathrm{c} / \mathrm{cw}$ direction. Furthermore, the $\mathrm{cw}$ rotation was also observed in the plasma biased using a hot cathode electrode. These experimental results suggest the ability of producing plasma poloidal rotations driven by rotating islands, although the island rotation was affected by the rotation of the target plasma. We can expect higher poloidal rotation velocity by increasing the frequency of the perturbation coil current.

\section{Acknowledgment}

This study was supported by a Grant-in-Aid from the Ministry of Education, Science and Culture of Japan (No. 17360439).

[1] T. Shimozuma et al., Nucl. Fusion 45, 1396 (2005).

[2] K. Ida et al., Nucl. Fusion 45, 391 (2005).

[3] M. Yakovlev, S. Inagaki et al., Phys. Plasmas 12, 092506 (2005).

[4] K. Ida, S. Inagaki et al., Phys. Plasmas 11, 2551 (2004).

[5] K. Ida, S. Inagaki et al., Nucl. Fusion 44, 290 (2004).

[6] A. Komori et al., Phys. Plasmas 8, 2002 (2001).

[7] S. Inagaki et al., Jpn. J. Appl. Phys. 36, 3697 (1997).

[8] S. Kitajima et al., Nucl. Fusion 46, 200 (2006).

[9] H. Takahashi et al., Plasma Phys. Control. Fusion 48, 39 (2006).

[10] S. Kitajima et al., Fusion Sci. Technol. 50, 201 (2006).

[11] S. Kitajima et al., Jpn. J. Appl. Phys. 30, 2606 (1991).

[12] S. Kitajima et al., J. Plasma Fusion Res. 4, 391 (2001).

[13] Y. Tanaka et al., Plasma Fusion Res. 2, S1019 (2007).

[14] Y. Tanaka et al., Plasma Phys. Control. Fusion 48, A285 (2006).

[15] Y. Tanaka et al., Trans. Fusion Sci. Technol. 51, 265 (2007). 